\title{
Surgical Treatment of Retrosternal Goitre
}

\author{
Kiera Welman ${ }^{1} \cdot$ Richard Heyes $^{2}\left([) \cdot\right.$ Paras Dalal $^{1} \cdot$ Sarah Hough $^{1} \cdot$ Marciano Bunalade $^{1} \cdot$ Vladimir Anikin $^{1}$
}

Received: 24 January 2016/Accepted: 27 June 2017/Published online: 4 July 2017

(C) Association of Otolaryngologists of India 2017

\begin{abstract}
This study aims to evaluate surgical approaches to the management of retrosternal goitre. Between 2004 and 2014, 35 patients (eight males; mean age $67.4 \pm 10.9$ years) with retrosternal goitre (mainly rightsided in 9, left-sided in 14 and bilateral in 12) underwent surgery. A palpable neck mass was found in 11 (31.4\%), stridor in $10(28.6 \%)$ and thyrotoxicosis in $4(11.4 \%)$ cases. $4(11.4 \%)$ patients were asymptomatic. Tracheal compression was detected radiologically in 27 (77.2\%) patients with deviation in $18(51.4 \%)$. A collar incision was performed in 34 patients, $6(17.1 \%)$ of whom required additional sternotomy, 1 (2.9\%) was assisted by an anterior mediastinotomy. 1 (2.9\%) had a right lateral thoracotomy. There was no operative mortality. Transient vocal changes occurred in $3(8.6 \%)$ patients, recurrent laryngeal nerve palsy in 3 , atrial fibrillation in 2 , and wound complications in $2(5.7 \%)$. Hospital stay ranged from 2 to 12 days $(5.5 \pm 2.0)$. Multinodular goitre was found in 33 patients, diffuse goitre in 1 and ectopic thyroid in 1 . The average vertical length of goitres in the
\end{abstract}

Presented at The 39th Biennial World Congress of the International College of Surgeons, Bali, Indonesia, October 20-26, 2014.

Kiera Welman

kiera.welman04@imperial.ac.uk

1 Department of Thoracic Surgery, The Royal Brompton and Harefield Hospital NHS Foundation Trust, Harefield Hospital, Hill End Road, Harefield, Middlesex, London UB9 6JH, UK

2 Department of Otolaryngology, Head and Neck Surgery, London North West Healthcare NHS Trust, Northwick Park Hospital, London, UK collar incision group was $7.6 \mathrm{~cm}$ compared to $10.6 \mathrm{~cm}$ in the sternotomy group. The average weight of specimens was $156.3 \mathrm{~g}$ in patients with collar incisions and $307.5 \mathrm{~g}$ in the sternotomy group. Removal of retrosternal goitre is more commonly performed via a cervical collar incision with mandatory availability of sternotomy. Radiological measurement of craniocaudal length may predict the risk of sternotomy. Surgical outcomes are not affected by surgical approach.

Keywords Retrosternal goitre $\cdot$ RSG $\cdot$ Goitre $\cdot$ Substernal Sternotomy

\section{Introduction}

Retrosternal goitre (RSG) is defined as a thyroid mass that extends 3 or more centimetres below the suprasternal notch when the neck is hyperextended [1]. Retrosternal extension can occur in 1-20\% of goitres, depending on the chosen definition [2]. RSG are usually benign in nature, although an underlying lymphoma or other neoplastic process may be found in up to $20 \%$ of cases [1]. Surgical removal is the gold standard curative treatment which prevents potentially fatal compressive symptoms. There are several surgical approaches, such as a collar incision, sternotomy, and thoracotomy. Some centres are currently evaluating the use of endoscopic techniques to assist surgical removal [3]. The aim of our study is to define the preoperative characteristics of patients with RSG, to evaluate the factors that result in particular surgical approaches, to assess clinical outcomes, and to optimise the treatment strategy. The intention of this study is to improve preoperative planning and better inform patients on an individual basis about the expected outcomes of surgery. 


\section{Patients and Methods}

Retrospectively analysis of the case files of all patients coded as having treatment for RSG in our department over a ten-year period from 2004 to 2014 was performed. Data were collected in line with the trust's policy on data protection, and data such as patient demographics, presentation, intraoperative management and follow-up details were recorded. All patients underwent computed tomography (CT) scanning prior to referral. Operations were performed by one of 3 consultant surgeons using similar techniques. Ultrasound and magnetic resonance imaging were not commonly performed before surgery because they offer little value to the assessment. A single radiology specialist analysed all radiological data, to avoid reporter bias. Simple data analysis techniques were used. There were no exclusions.

There were 35 patients referred electively to our centre in the study period. The age range was $37-87$ years, with a mean age of $67.4 \pm 10.9$ years. There were $27(77 \%)$ females and $8(23 \%)$ males. Only $9(25.7 \%)$ had right-sided pathology. In 4 (11\%) patients, the RSG was detected incidentally on routine chest radiography; these patients had no relatable signs or symptoms. The other 31 patients had a combination of symptoms, the most common being breathing difficulties such as occasional stridor and shortness of breath on exertion (Table 1). The second most common symptom was dry cough, usually of several months' duration. This often occurred in conjunction with breathing difficulties, but in $3(8.5 \%)$ patients, dry cough was the only symptom experienced. Vocal changes were noted in $10(28.6 \%)$ cases, mostly comprising deepening or weakening of the voice. Only one of these patients had proven complete recurrent laryngeal nerve palsy preoperatively. Other presenting complaints included acid reflux, dysphagia and chest pain (Table 1). One patient had the unusual presentation of right-sided ear pain. Initial examination revealed a palpable anterior neck mass in 11 $(31.4 \%)$, stridor in $10(28.6 \%)$ and dilated neck veins in 6 (17.1\%; Table 2). The majority of patients were euthyroid,

Table 1 Clinical symptoms in 35 patients with retrosternal goitre

\begin{tabular}{llc}
\hline Symptom & No. of cases & Percentage \\
\hline Exertional dyspnoea & 22 & 62.9 \\
Dry cough & 11 & 31.4 \\
Vocal changes & 10 & 28.6 \\
Acid reflux & 8 & 22.9 \\
Dysphagia & 5 & 14.3 \\
Chest pain & 3 & 8.6 \\
Referred otalgia & 1 & 2.9 \\
Asymptomatic & 4 & 11.4 \\
\hline
\end{tabular}

but $4(11.4 \%)$ had evidence of thyrotoxicosis and one was hypothyroid.

The pathology was predominantly left-sided $(n=14$, $40 \%)$ or bilateral $(n=12,34.3 \%)$. Radiological evidence of tracheal compression (Fig. 1) was seen in $27(77.2 \%)$ patients, and tracheal deviation was seen in $18(51.4 \%)$. In most cases, the tracheal deviation was contralateral to the predominant side of the pathology, however, in one patient with bilateral pathology, the deviation was apparently posterior with displacement of the oesophagus laterally. Superior vena cava (SVC) compression was evident on the CT scans of 2 patients, both of whom had dilated neck veins on initial examination. The longest goitre recorded on $\mathrm{CT}$ was $18 \mathrm{~cm}$ in craniocaudal length, the shortest was $3.3 \mathrm{~cm}$. The widest measurement in both transverse and anteroposterior diameters was $8 \mathrm{~cm}$. In 21 patients, the lower edge of the goitre made contact with the aortic arch. In the majority of cases, most of the mass was contained within the anterior mediastinum, but 5 patients had RSG mainly in the posterior mediastinum and 8 had RSG in the superior mediastinum. Five patients also underwent preoperative radiology-assisted biopsies of the RSG, which were all reported as Thy-2 in nature.

Thirty-four patients underwent an cervical collar incision. Six of whom (17.6\%) also underwent a sternotomy and $1(2.9 \%)$ had the assistance of a right anterior mediastinotomy (Fig. 2). 1 patient (2.9\%) was treated with a lateral thoracotomy incision without a collar incision. The right anterior mediastinotomy was employed to push the right-sided goitre from below to deliver it up and out through the collar incision; this technique avoided the need for sternotomy. A technique still utilised in our department, which may also prevent the need for sternotomy should the use of a finger through the cervical incision fail to deliver the thyroid, is the use of a sterilised dessertspoon to scoop the RSG mass from within its cavity and present it out through the collar incision (Fig. 3).

\section{Results}

There was no operative mortality. The mean anaesthetic time was $128.9 \mathrm{~min}$ in the cervical collar group and $225.8 \mathrm{~min}$ in sternotomy patients. All patients spent 1-2 days in the intensive therapy unit. Hospital stay ranged from 2 to 12 days (mean $5.5 \pm 2.0$ days). In the cervical collar group, mean hospital stay was 5 days, whereas in the sternotomy group, the mean stay was 7 days. The majority of patients had no complications postoperatively. The most common complication after surgery was vocal changes in 6 (17.1\%) patients, 3 of whom were found to have recurrent laryngeal nerve palsy; of these, 2 had a collar incision alone and the other underwent thoracotomy without collar 
Table 2 Clinical findings in 35 patients with retrosternal goitre

\begin{tabular}{llc}
\hline Finding & No. of cases & Percentage \\
\hline Stridor & 10 & 28.6 \\
Anterior neck mass & 11 & 31.4 \\
Dilated neck veins & 6 & 17.1 \\
Hypothyroidism & 1 & 2.9 \\
Hyperthyroidism & 4 & 11.4 \\
Empyema & 1 & 2.9 \\
Positional dyspnoea & 1 & 2.9 \\
\hline
\end{tabular}

incision. Only one patient with vocal changes had undergone a sternotomy and this had resolved at 1 month followup. Sternotomy was therefore not associated with increased risk of vocal changes. Other complications included postoperative atrial fibrillation and wound complications in 2 $(5.7 \%)$ patients each. Only $1(2.9 \%)$ patient had transient hypocalcaemia. No patient experienced tracheomalacia or needed a tracheostomy, and none experienced postoperative bleeding or haematoma. Overall, our complication rate was low and there was no evidence that any complication was more common following sternotomy. All 35 patients
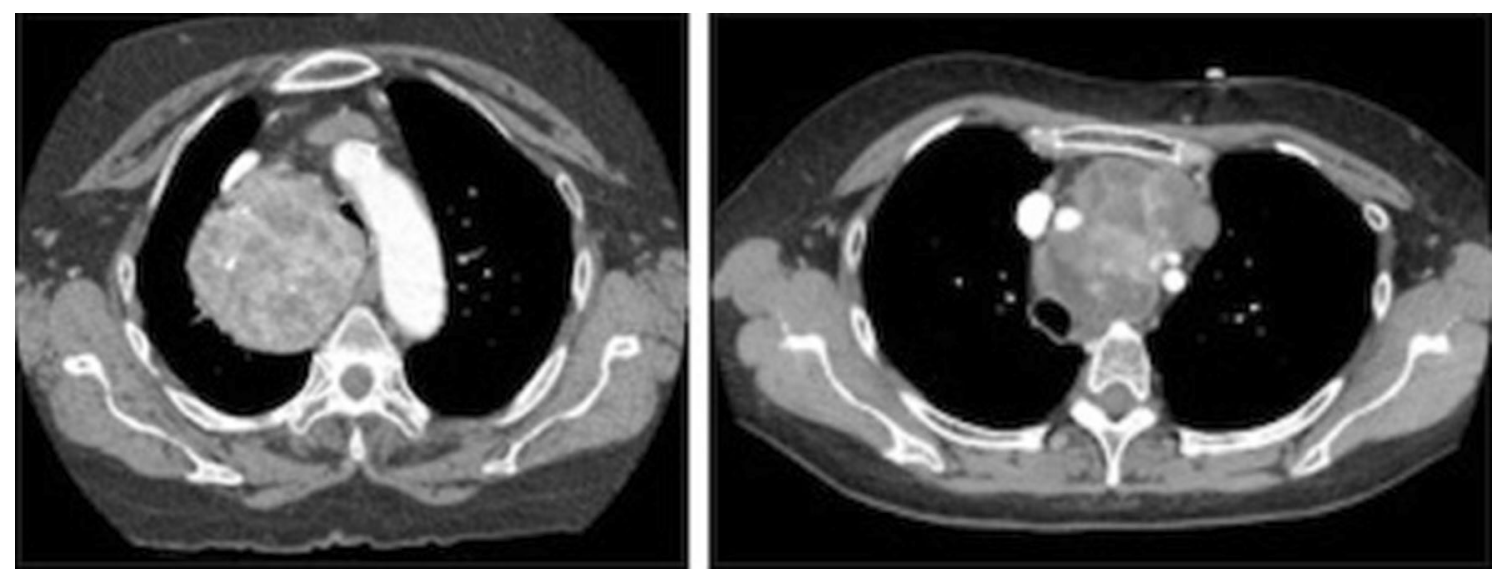

Fig. 1 Sections from contrast-enhanced computed tomography scans of the thorax in 2 patients showing: a severe tracheal compression, and b tracheal deviation
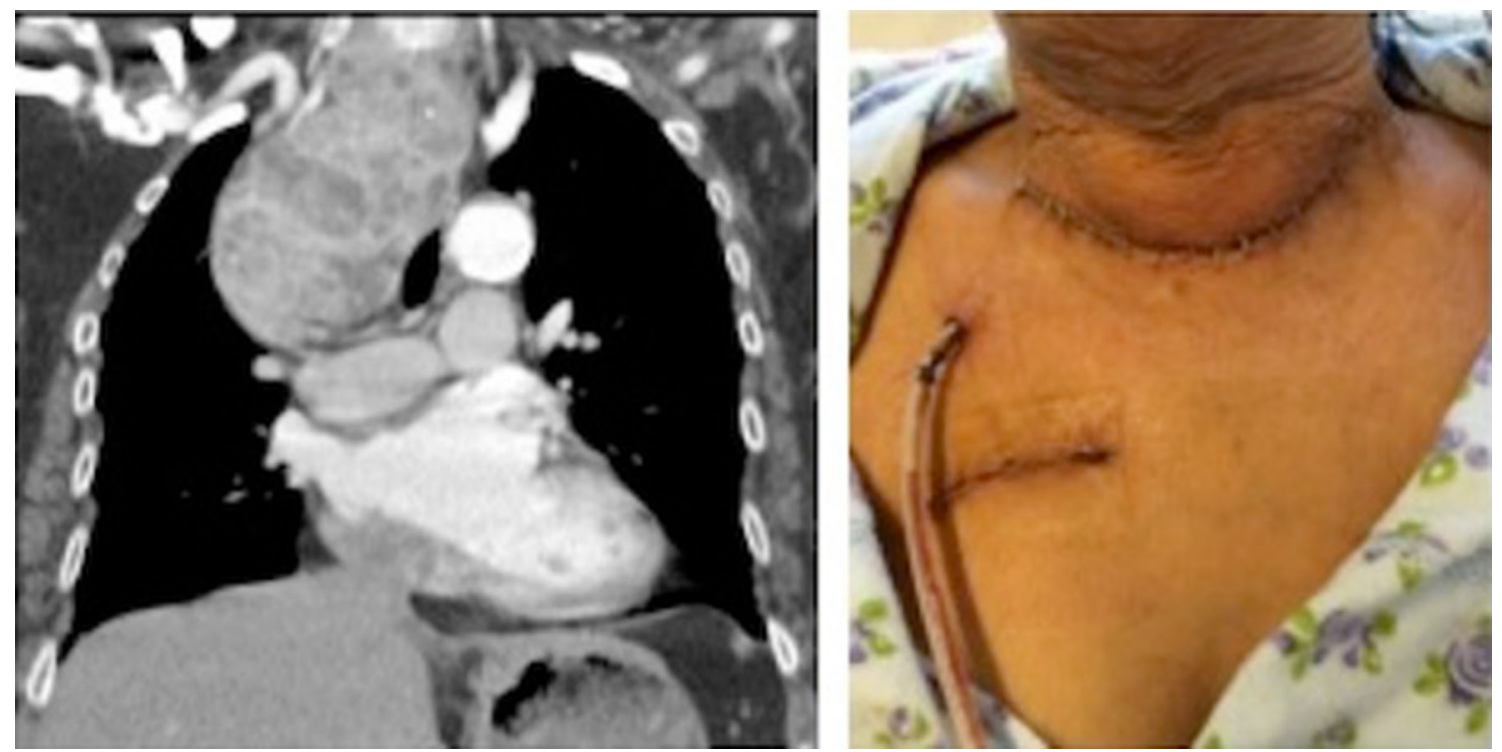

Fig. 2 Preoperative computed tomography a and postoperative photograph, b of a patient who underwent a collar and right anterior mediastinotomy incision 


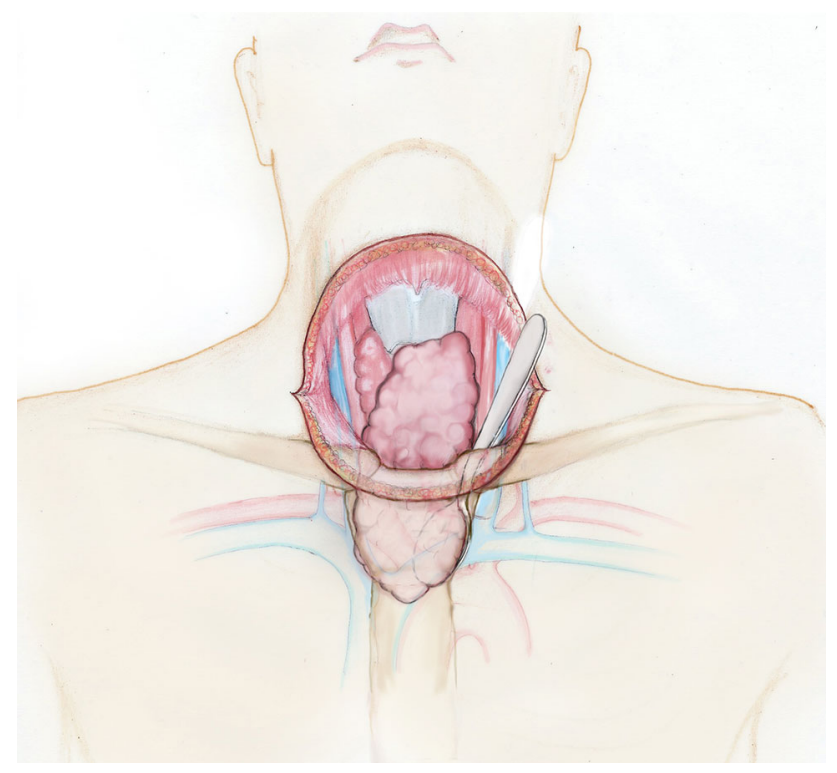

Fig. 3 The "spoon technique" to deliver the mass through a cervical incision

were followed up at 1 month postoperatively, and all except 4 reported a resolution of their original symptoms. Of these 4, 3 had ongoing breathing difficulties and 1 had worsening of her thyroid-related eye symptoms. No patient required a postoperative blood transfusion. The difference in pre- and post-operative haemoglobin levels was used as a surrogate marker for blood loss. The average difference in pre- to post-operative haemoglobin was $1.5 \mathrm{~g} / \mathrm{dL}$ in the cervical collar group versus $3.1 \mathrm{~g} / \mathrm{dL}$ in the sternotomy group.

All specimens were sent for histology. Thirty-three patients were diagnosed with multinodular goitre, 1 with smooth goitre, and 1 had ectopic normal thyroid tissue. A difference was seen in the weight of specimens obtained via cervical collar alone versus sternotomy, with the average specimen being $156.3 \mathrm{~g}$ in collar incisions and $307.5 \mathrm{~g}$ in sternotomy patients. Preoperative CT showed that the average vertical length of goitres in the collar incision group was $7.6 \mathrm{~cm}$, and the average length of goitres in the sternotomy group was $10.6 \mathrm{~cm}$. There was no correlation between goitre size and the number or type of symptoms experienced.

\section{Discussion}

Surgical treatment is the gold-standard management in patients with RSG. However, alternatives such as "watch and wait" may be considered because RSGs are typically slow growing in nature. In patients with evidence of airway compromise, most centres would strongly encourage operative management and some have successfully employed tracheobronchial stenting as a procedure to delay definitive surgery where appropriate [4].

Our patients were predominantly female, which is similar to the findings in other studies [1]. RSG detection as an incidental finding was $11 \%$, although is quoted to be up to $20 \%$ in some areas of the world [2]. The majority of patients $(68.6 \%)$ did not present with an anterior neck mass. The most common presenting complaint in our patients was breathing difficulties, secondary to tracheal deviation or compression. In one patient, the unusual presentation of right-sided ear pain occurred in association with a large left-sided RSG causing deviation of structures to the right. It is thought that this may have been referred pain created by pressure on the vagus nerve, and it is a rare phenomenon [5].

RSG is usually contained within the anterior mediastinum, however, posterior mediastinal RSG occurs in $10-15 \%$ of cases [6]. Most RSG can be managed through a cervical incision although it has been suggested that RSG with a large proportion of mass in the posterior mediastinum carry a higher risk of sternotomy which is more technically challenging and traumatic [7]. Extension of the mass to the aortic arch and SVC obstruction are also factors that have been attributed to increased rates of sternotomy $[8,9]$. Five $(14.2 \%)$ of our patients had RSG mainly in the posterior mediastinum. All were managed with a collar incision and the "spoon technique". We anticipate that this technique might provide better protection to the recurrent laryngeal nerve, which can be difficult to identify intraoperatively, and often has a close relationship to the goitre outside the capsule. A similar technique has been described previously using a Parker-Langenbeck retractor to deliver the RSG through a collar incision [10].

All six patients who underwent sternotomy had evidence of extension to the level of the aortic arch. Overall, 21 of the 35 patients in our study had extension of the RSG to the aortic arch. Within our practice, $28 \%$ of RSG that involve the aortic arch require a sternotomy, and therefore aortic arch extension is rejected as a reliable predictor of sternotomy. Of two patients with evidence of SVC obstruction on preoperative $\mathrm{CT}$, one underwent a collar incision alone without sternotomy which demonstrates that SVC obstruction does not imply obligatory sternotomy.

The average vertical length of goitres in the collar incision group was $7.6 \mathrm{~cm}$, whereas the average length of goitres in the sternotomy group was $10.6 \mathrm{~cm}$. In the average-sized person, those who require sternotomy for surgical removal tend to have an RSG that is $3 \mathrm{~cm}$ longer in craniocaudal dimension than those who can be managed by collar incision alone. Therefore the craniocaudal length of the goitre is possibly a more reliable predictor of sternotomy in our practice. 
There was no operative mortality among our patients, however, previous studies have reported mortality rates of up to $2.3 \%$ [11]. Overall complication rate was low and vocal changes were the most common adverse effects $(17.1 \%)$. The rate of recurrent laryngeal nerve palsy in our patients was $8.5 \%$, which compares favourably with previously reported rates of up to $13.2 \%$ [9]. In thyroid surgery without retrosternal extension, the rate of recurrent laryngeal nerve palsy is widely accepted to be less than $1 \%$. It is well-documented that retrosternal extension carries a greater risk of injury to the recurrent laryngeal nerve during surgical removal [12]. However, despite the occurrence of pulmonary complications, one group have reported a lower incidence of recurrent laryngeal nerve injury when employing sternotomy as an approach over cervical approach [13].

Another widely reported complication is transient hypocalcaemia, occurring in up to $6 \%$ of surgeries for RSG secondary to parathyroid manipulation or damage [14]. This complication was encountered in only one $(2.8 \%)$ patient. There was no variation in length of intensive care stay by surgical approach, but in sternotomy cases the average anaesthetic time was 103 min longer and hospital stay was 2 days longer. No complication was more common with sternotomy.

Surgical management for patients with RSG requires careful preoperative planning and many factors should be taken into account when considering alternative approaches such as sternotomy and thoracotomy. Manubriotomy is considered to be another safe alternative, which allows exposure of structures within the thoracic inlet although direct comparison of this technique against others has not been evaluated in terms of patient risk [12]. Newer techniques such as endoscope-assisted and video-assisted thoracoscopic thyroidectomy also provide options for minimally invasive surgical removal of RSG [15]. Further study of the success of these techniques is needed.

Age discrimination in surgery is a widely debated topic in the United Kingdom. The Royal College of Surgeons have reported on access to surgery in relation to age and suggest that biological rather than chronological age should be considered clinically relevant [16]. The oldest patient operated on in our study was 87-years old and the mean age of our patients (67.4 years) is older than the mean ages of 40-50 years quoted in other studies [1].

\section{Conclusion}

Patients requiring removal of RSG are typically female with a mean age of 67.4 years. The main complaint caused by RSG is breathing difficulties, and clinical diagnosis may be hindered by the lack of an anterior neck mass. Surgery is more commonly performed via a cervical collar incision with mandatory availability of additional sternotomy. Sternotomy is a longer and technically more difficult procedure, however, the need for this approach is relatively low even in the face of posterior mediastinal involvement, aortic arch extension, or SVC compression, which are commonly thought to be predictors of sternotomy. Instead, we advocate that craniocaudal length is a more reliable predictor of the need for sternotomy because sternotomy patients have goitres $3 \mathrm{~cm}$ longer than those who undergo collar incision alone. Our favourable outcomes may also be due in part to the intraoperative techniques employed, such as the "spoon technique". As a result of this study, we feel better placed to advise our patients that should they need to undergo sternotomy, there is no increased rate of complications and hospital stay is usually only 2 days longer than that of the average collar-incision patient. Due to the limited sample size, further data collection is needed to strengthen these conclusions.

\section{Compliance with Ethical Standards}

Conflict of interest The author(s) declare no potential conflicts of interest with respect to the research, authorship, and/or publication of this article.

Ethical Approval All procedures performed in studies involving human participants were in accordance with the ethical standards of the institutional and/or national research committee and with the 1964 Helsinki declaration and its later amendments or comparable ethical standards. This article does not contain any studies with animals performed by any of the authors.

Informed Consent Informed consent was obtained from all individual participants included in the study.

\section{References}

1. Coskun A, Yildirim M, Erkan N (2014) Substernal goiter: when is a sternotomy required? Int Surg 99:419-425

2. Rodrigues J, Furtado R, Ramani A, Mitta N, Kudchadkar S, Falari S (2013) A rare instance of retrosternal goitre presenting with obstructive sleep apnoea in a middle-aged person. Int J Surg Case Rep 12:1064-1066

3. Fan Q, Gong K, Zhu B, Zhang NW (2014) Experience of managing substernal goitre by totally endoscopic procedure. Beijing Da Xue Xue Bao 46:488-491

4. Piao M, Yuan Y, Wang Y, Feng C (2013) Successful management of trachea stenosis with massive substernal goitre via tracheobronchial stent. J Cardiothorac Surg 8:212

5. Schoenen J, Broux R, Moonen G (1992) Unilateral facial pain a the first symptom of lung cancer: are there diagnostic clues? Cephalalgia 12:178-179

6. Kacprzak G, Karas J, Rzechonek A, Blasiak P (2012) Retrosternal goitre located in the mediastinum: surgical approach and operative difficulties. Interact Cardiovasc Thorac Surg 15:935-937

7. Chen X, Xu H, Ni Y, Sun K, Li W (2013) Complete excision of a giant thyroid goiter in posterior mediastinum. J Cardiothorac Surg 8:207 
8. McKenzie GA, Rook W (2014) Is it possible to predict the need for sternotomy in patients undergoing thyroidectomy with retrosternal extension? Interact Cardiovasc Thorac Surg 19:139-143

9. Sakkary MA, Abdelrahman AM, Mostafa AM, Abbas AA, Zedan MH (2012) Retrosternal goitre: the need for thoracic approach based on CT findings: surgeon's view. J Egypt Natl Canc Inst 24:85-90

10. Naraynsingh V, Ramarine I, Cawich S, Maharaj R, Dan D (2013) Cervical leverage: a new procedure to deliver deep retrosternal goitres without thoracotomy. Int J Surg Case Rep 11:992-996

11. Landerholm K, Järhult J (2014) Should asymptomatic retrosternal goitre be left untreated? A prospective single-centre study. Scand J Surg 23:1-4
12. Testini M, Gurrado A, Bellantone R, Brazzarola P, Cortese R, De Toma $G$ et al (2014) Recurrent laryngeal nerve palsy and substernal goiter. An Italian multicenter study. J Visc Surg 151:183-189

13. Rolighed L, Rønning H, Christiansen P (2015) Sternotomy for substernal goiter: retrospective study of 52 operations. Langenbecks Arch Surg 400:301-306

14. Shaha AR, Jaffe BM (1998) Parathyroid preservation during thyroid surgery. Am J Otolaryngol 19:113-117

15. Gupta P, Lau KK, Rizvi I, Rathinam S, Waller DA (2014) Video assisted thoracoscopic thyroidectomy for retrosternal goitre. Ann R Coll Surg Engl 96:606-608

16. Ahamat N (2012) Access all ages: assessing the impact of age on access to surgical treatment. Bull R Coll Surg Engl 94(9):300 